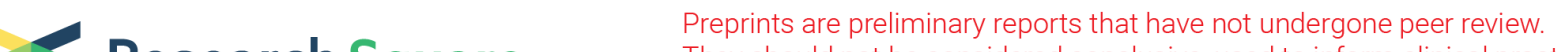 Research Square They should not be considered conclusive, used to inform clinical practice, or referenced by the media as validated information.
}

\section{An integrated analysis of the competing endogenous RNA network associated of prognosis of stage I lung adenocarcinoma}

\section{Yuan Xu}

Second Affiliated Hospital of Fujian Medical University

\section{Guofu Lin}

Second Affiliated Hospital of Fujian Medical University

Yifei Liu

Second Affiliated Hospital of Fujian Medical University

Xianbin Lin

Second Affiliated Hospital of Fujian Medical University

Hai Lin

Second Affiliated Hospital of Fujian Medical University

\section{Zhifeng Guo}

Second Affiliated Hospital of Fujian Medical University

Yingxuan Xu

Second Affiliated Hospital of Fujian Medical University

Qinhui Lin

Second Affiliated Hospital of Fujian Medical University

Shaohua Chen

Second Affiliated Hospital of Fujian Medical University

Jiansheng Yang

Second Affiliated Hospital of Fujian Medical University

Yiming Zeng ( $\boldsymbol{\nabla}$ zeng_yiming@fjmu.edu.cn )

Second Affiliated Hospital of Fujian Medical University

\section{Research Article}

Keywords: lung adenocarcinoma, stage I, IncRNA, ceRNA, FENDRR

Posted Date: February 19th, 2021

DOI: https://doi.org/10.21203/rs.3.rs-208860/v1 
License: (c) (i) This work is licensed under a Creative Commons Attribution 4.0 International License. Read Full License

Version of Record: A version of this preprint was published at BMC Cancer on February 19th, 2022. See the published version at https://doi.org/10.1186/s12885-022-09290-0. 


\section{Abstract}

Background: Accumulating evidence indicates that long non-coding RNAs (IncRNAs) are involving in the tumorigenesis and metastasis of lung cancer. The aim of the study is to systematically characterize the IncRNA-associated competing endogenous RNA (ceRNA) network and identify key IncRNAs in the development of stage I lung adenocarcinoma (LUAD).

Methods: Totally, 1,955 DEmRNAs, 165 DEmiRNAs and 1,107 DElncRNAs were obtained in 10 paired normal and LUAD tissues. And a total of 8,912 paired IncRNA-miRNA-mRNA network was constructed. Using the Cancer Genome Atlas (TCGA) dataset, the module of ME turquoise was revealed to be most relevant to the progression of LUAD though Weighted Gene Co-expression Network Analysis (WGCNA).

Results: Of the IncRNAs identified, LINC00639, RP4-676L2.1 and FENDRR were in ceRNA network established by our RNA-sequencing dataset. Using univariate Cox regression analysis, FENDRR was a risk factor of progression free survival (PFS) of stage I LUAD patients (HRs $=1.69,95 \% \mathrm{Cl} 1.07-2.68, P<.050$ ). Subsequently, differential expression of FENDRR in paired normal and LUAD tissues was detected significant by real-time quantitative (qRT-PCR) $(P<0.001)$.

Conclusions: This study, for the first time, deciphered the regulatory role of FENDRR/miR-6815-5p axis in the progression of early-stage LUAD, which is needed to be established in vitro and in vivo.

\section{Introduction}

Lung cancer remains the prominent contributor of cancer-related mortality, with the worldwide 5-year survival rate of which is around $16.6 \%[1,2]$. Recently, with the wide application of low-dose computed tomography for early screening and the rapid development of target drugs for genetic mutations, the progress against lung cancer has achieved profound success. As of 2017, the mortality rate of lung cancer dropped from its peak by $51 \%$ among males and by $26 \%$ among females [2]. Nonetheless, the 5year survival rate for lung cancer patients is still not well manifested. Additionally, the early-stage patients' prognosis displays quite disparate from those of advanced-stage, with 5-year survival ranging from $85 \%$ to $6 \%$ [3].

Currently, non-small cell lung cancer (NSCLC), accounting for approximately $85 \%$ of all lung cancer, has been endowed with several therapeutic options, including surgery, chemotherapy, radiation, target therapy and immunotherapy [4]. And surgery is the first choice of curative treatments for the medically operable. Owing to its readily enters into regional lymph nodes and apt to metastasize at an early stage, however, the recurrence rate accounts for approximately $27 \%$ to $38 \%$ for stage I NSCLC patients [5-7]. Therefore, intensive efforts have been directed to elucidate the molecular mechanism of premalignancy development and progression, and to identify potential molecular signatures for early diagnosis and interception. 
Recently, long noncoding RNAs (IncRNAs) have attracted significant attentions in various cancers. LncRNA is a class of transcripts with length of more than 200 nucleotides that possesses limited or no protein-coding capacity, which is transcribed by RNA polymerase II, spliced, 5'capped, and polyadenylated[8]. It has been identified that IncRNAs were involved into diverse cellular, physiological and pathological process via a series of mechanism $[9,10]$, including serving as critical regulators of tumorigenesis and metastasis [11]. Furthermore, accumulating evidences revealed that IncRNA could disrupt miRNA-mediated degradation of target mRNAs by acting as "miRNA sponges" [12], indicating coding and noncoding RNAs could control one another through their ability to compete for miRNA binding locus, which termed as "ceRNA". Under this hypothesis, an increasing amount of evidences revealed that ceRNA axis could contribute to tumorigenesis, progression and metastasis of cancer [13, 14]. For instance, researchers have demonstrated that $L N C 00336$, an novel regulator of ferroptosis, could act as a ceRNA to mediate the expression of cystathionine- $\beta$-synthase (CBS) by sponging miR6852, which may serve as an potential therapeutic target of lung cancer [15]. Also, a novel IncRNA AFAP1-AS1 was reported to be involved in the development and progression of NSCLC via up-regulating interferon regulatory factor (IRF)7 by retinoid-inducible protein (RIG)--like receptor signaling pathway [16]. However, most of previous studies on IncRNAs acting as ceRNAs were carried out on NSCLC patients with ignoring the heterogeneity of staging, and the clinical significance of tumor progression in early stage remains largely unknown.

Lung adenocarcinoma (LUAD) accounts for the most common subtype of NSCLC, which brings to light the necessity of distinguishing LUAD from other subtypes at an early stage to propose individual treatment. To date, it has been revealed that EGFR and KRAS mutations, and multiple other oncogenic and tumor suppressor genes were involved in the process of initiation and pathogenesis $[17,18]$. Given tumor's biological behaviors and molecular characteristic displaying heterogeneous at an early stage, some patients could experience a long-term survival, while others could not. To address this, we performed RNA-sequencing for stage I LUAD patients to identify differentially expressed IncRNAs (DElncRNAs) in paired normal and tumor tissues, following by ceRNAs network constructed. On this basis, IncRNA-related ceRNAs identified were validated by database from The Cancer Genome Atlas (TCGA) to establish clinical prognostic model. Furthermore, the hub gene related ceRNAs regulatory network of IncRNAs-miRNAs-mRNAs were established to broaden our knowledge of expression pattern.

\section{Methods}

\section{Patients and clinical samples}

A total of 10 paired LUAD and adjacent normal fresh tissues were obtained from Second Affiliated Hospital of Fujian Medical University between January, 2019 and May, 2019. The specimens were collected from LUAD patients with treatment-naïve who underwent primary surgical treatment. All of the specimens were immediately snap-frozen by liquid nitrogen after surgical resection, and stored at $-80^{\circ} \mathrm{C}$ until RNA extraction. The clinicopathological diagnosis were confirmed by two pathologists according to the guidelines of the World Health Organization (WHO, version 2015). All participants provided written 
informed consent, and the bioethical committees at The Second Affiliated Hospital of Fujian Medical University, China, gave written approval for the study (2020-206).

The other group resulted from the LUAD-related RNA-sequencing data in TCGA database, containing the expression data of mRNAs, miRNAs and IncRNAs. A total of 595 paired LUAD tissues RNA-seq data were retrieved, with their clinicopathological features. RNA-sequencing data and corresponding clinical information in LUAD were obtained from TCGA database as following criteria: 1) histologically diagnosed as LUAD; 2) data with complete clinical information; 3 ) diagnosed as stage I patients. Finally, a total of 260 paired LUAD tissues was included for further analysis. The present study meets the criteria of data usage and publishing of the National Cancer Institute of $\mathrm{NIH}$, and no approval from the ethics committee was required. Progression-Free Survival (PFS) was calculated from the date when patients first received treatment until the date of progression or the last follow-up or death.

\section{RNA extraction and sequencing}

Total RNA of LUAD tissues and corresponding normal tissues was extraction from frozen tissues using RNeasy Mini Kit (Qiagen, Germany) following the manufacturer's protocol. The RNA concentration was evaluated by the Qubit 4.0 (Thermo Fisher Scientific, Wilmington, DE, USA), and the RNA quality was evaluated by agarose gel eletrophoresis.

Then, ribosomal RNA was removed from total RNA to obtain the maximum residual ncRNA. After fragment of rRNA-depleted RNA, the cDNA library was constructed using the TruSeq RNA sample Prep Kit (Illumina, San Diego, CA, USA). LncRNA/mRNA sequencing libraries were prepared using VAHTS total RNA-seq Library Prep kit for Illumina (Vazyme NR603, China) according to the manufacturer's protocol. The cDNA fragments with 150-bp paired-end reads were generated for RNA sequencing. Additionally, NEBNext ${ }^{\circledR}$ Multiplex Small RNA Library Prep Set for Illumina ${ }^{\circledR}$ (NEB) was used to prepare the miRNA library for samples. 12 libraries were pooled and sequenced in a single lane of Illumina HiSeq Xten sequencing platform. And Illumina's TruSeq small RNA library preparation kit was used to prepare the miRNA library with 50-bp paired-end reads generated. After library construction, RNA sequencing for both IncRNA/mRNA and miRNA was performed using Illumina HiSeq Xten platform.

\section{Identifications of DEmRNAs, DEmiRNA and DElncRNAs}

Mirdeep2 (v2.0.0.5) was used for new miRNA prediction, whose expression was calculated and standardized using CPM (counts per million read, CPM). LncRNAs were annotated by the following database, including PLEX (https:// sourceforge.net/projects/plek/), CPAT

(https://sourceforge.net/projects/rna-cpat/), CNCl (https://github.com/www-bioinfo-org/CNCl) and CPC2 (http:// cpc2.cbi.pku.edu.cn/). The intersection was exhibited using Venn diagram. (Fig. S1).

DESeq2Rpackage(https://bioconductor.org/packages/release/bioc/html/DESeq2.html) in Bioconductor project was used to screen the differentially expressed mRNA (DEmRNAs), differentially expressed miRNA (DEmiRNAs) and differentially expressed IncRNA (DElncRNAs) between LUAD and normal tissues. 
$\mid \log _{2}$ (fold change)| $\left(\mid \log _{2} \mathrm{FCl}\right) \geq 1$ and statistical $P$ value $\leq 0.05$ were set as cut-off criteria. Finally, unsupervised hierarchical clustering was carried out for DE-RNAs, and the expression patterns of which in paired tissues were displayed in form of heatmap using the pheatmap R package (https://cran.rproject.org/web/ packages/pheatmap/index.html).

\section{Analysis of the DElncRNAs Enrichment pathway}

Gene ontology (GO, http://www.bioconductor.org/packages/release/bioc/ html/topGo.html) analysis was conducted to screen enrichment of target genes to determine the biological functions regulated by IncRNAs, including biological processes (BP), cellular component (CC), and molecular function (MF) annotations. Kyoto Encyclopedia of Genes and Genomes (KEGG, http://www.genome.jp /keggbin/show_organism?menu_type=pathway_maps\&org=hsa) analysis was performed to annotate the signaling pathways mainly involved for target genes. The GO and pathway analysis with enriched gene count $\geq 2$ and $P$ value $<0.05$ as the threshold for statistical significance.

\section{Predication of miRNA Regulation Relationship}

The predication of miRNA-gene analysis of DEmiRNA obtained was performed using the online website miRWalk 2.0 (http://zmf.umm.uni-heidelberg.de/ pps/zmf/mirwalk2/). Furthermore, miRWalk, miRanda, miRDB, miRMap and TargetScan databases were used to predict the possible DEmiRNA-DEmRNA regulatory relationships. Using the StarBase (http://starbase.sysu.edu.cn/) database, the miRNA-IncRNA regulatory relationships by DEmiRNA were predict. Then the DEmRNA-DEmiRNA and DEmiRNA-DEIncRNA regulatory regulation relationship were constructed based on shared miRNAs with which both IncRNAs and mRNAs interact, and were illustrated by Cytoscape software.

\section{Construction of IncRNA-miRNA-mRNA ceRNA network}

Under the hypothesis of miRNA sponge, the positive correlation expression of IncRNAs-mRNAs was focused on, therefore, and the positive correlation coexpression relationship between mRNAs and IncRNAs simultaneously regulated by miRNAs was obtained. ceRNA network were constructed based on shared miRNAs with which mRNAs and IncRNAs interact. For further analysis, we used a hypergeometric cumulative distribution function test to determine potential ceRNA pairs[19], with correlation coefficient $>0.5$ and $P$ value $<0.05$ as a ceRNA triplet with statistical significance.

\section{Weighted gene correlation network analysis (WGCNA)}

As described previously [20,21], gene co-expressed network analysis was performed on LUAD and adjacent normal tissues using R WGCNA package. The expression matrix was restricted to expressed IncRNAs, following by a Pearson correlation matrix and a weighted adjacency matrix generated. The module eigengene (ME) was calculated for each module, the values of which clinical traits associated with was calculated by Pearson's correlation. In the present study, we set the optimal soft-thresholding power at 4 (scale-free $R^{2}=0.85$ ), cut height at 0.25 , and minimal modules sized to 30 , to identify key 
modules. topological overlap matrix (TOM) was constructed and transformed. Then, the IncRNAs were grouped into co-expression modules using tree pruning of gene hierarchical clustering dendrograms by cutreeDynamic method, with correlated modules merged. The module significantly correlated with sample traits were used for hub genes selection.

\section{LUAD-specific prognostic IncRNA signatures identification}

Progression-free survival (PFS) time was calculated from the date when patients first received chemotherapy until the date of progression or the last follow-up or death. Using TCGA dataset, the associations between DElncRNAs in ceRNA network and PFS in stage I LUAD patients were evaluated using univariate Cox regression analysis. The samples were divided into high expression and low expression groups based on the median FPKM of IncRNAs. Kaplan-Meier (KM) survival analysis and Logrank (LR) test were performed, and LR P value, hazard ratio (HR) with $95 \%$ confidence interval (CI) were computed. Survival and survminer $\mathrm{R}$ package were used for Cox regression analysis, and the statistical $P<0.05$ was considered as significant.

\section{RNA extraction and quantitative real-time RT-PCR assay}

Total RNA was extracted using (Trizolß; Sigma) from 28 paired LUAD tissues, according to the manufacturer's recommendations. After purification, the RNA was transcribed into cDNA using Reverse Transcription Kit (Takara, Tokyo, Japan). RT-PCR analysis was performed using the SYBR Green (Takara). The primers used for RT-PCR were: FENDRR primers were AGTCACAGCACCAGAAAGCCAAC (sense) and TGATGTTCTCCTTCTT GCCTCAGC (anti-sense); LNC00639 primers were GTGAGTGTTCAGACATGCCAGGAG (sense) and AGCCGAGTGGATTCAGCGAGAG (anti-sense); RP4676L2.1 primers were TGTTTGAAGCCGTGAGACTGAGTG (sense) and CTCCTTTGCTGGCTC TTCCTCATC (anti-sense).

\section{Results}

\section{Identification of DEmRNAs, DEmiRNAs and DElncRNAs}

The schematic diagram was constructed to display the methods of the present study (Figure 1). Initially, to explore the role of IncRNAs in stage I LUAD, we performed RNA-seq analysis of total RNA in 10 paired tumor and adjacent normal tissues. According to the criteria of $\left|\log _{2} \mathrm{FC}\right| \geq 1$ and $P$ value $\leq 0.05$ cut-off, a total of 1,955 DEmRNAs (1,372 up- and 583 down-regulated), 165 DEmiRNAs (111 up- and 54 downregulated) and 1,107 DElncRNAs (775 up- and 332 down-regulated) were detected from dataset. The heatmaps of DEmRNAs, DEmiRNAs and DElncRNAs were presented in Figure 2A-C, and the top 20 of which were displayed in Table S1-3, respectively.

To explore the significance of DElncRNAs that might involve in LUAD, we performed functional enrichment analysis of target mRNAs. It implied that the most significant BP, CC and MF by DElncRNAs enriched were embryo development (G0:0009790, $P=3.10 \mathrm{E}-09)$, presequence translocase-associated 
import motor (G0:0001405, $P=0.0002)$ and nucleic acid binding transcription factor activity (G0:0001071, $P=6.10 \mathrm{E}-07$ ), respectively. Figure 2D displayed the top $20 \mathrm{GO}$ terms enriched by DElncRNAs. Additionally, pathway enrichment analysis was performed to reveal potential biological function of DElncRNAs. It showed that the top 20 pathways were significantly enriched using Kyoto Encyclopedia of Genes and Genomes (KEGG) analysis, with glycerophospholipid metabolism pathway enriching the most significant genes in the network (Figure 2E,2F).

\section{ceRNA network construction}

With the criteria of total score $\geq 150$ and total energy $\leq-20$, we used miRanda to predict the regulation relationship between miRNA and mRNA, as well as the relationship between miRNA and IncRNA (Figure $3 A, 3 B)$. According to the regulation of DElncRNA-DEmiRNA and DEmRNA-DEmiRNA, IncRNAs and mRNAs regulated by the same miRNA were screened. Using the parameter of Pearson correlation (PPC) $>0.5$ and $P$ value $<0.05$ as a threshold, a total of 8,912 interaction relationships of IncRNA-miRNA-mRNA with statistical significance were predicted in 10 paired LUAD tissues, and the top 20 ceRNA network were listed in Table S4.

\section{Co-expression module identification and Hub gene selection}

To further explore the potential function of IncRNAs in the progression of LUAD, the transcriptomic data of 260 stage I LUAD patients and their corresponding clinical data in TCGA database were extracted to construct WGCNA network. In this study, we chose $\beta=4\left(R^{2}=0.85\right)$ as a soft threshold to construct a scalefree topology network (Figure 4A). The TOM including all genes was depicted by topological overlapping heat map. Totally, 43 gene co-expression modules were identified by linkage hierarchical clustering according to TOM-based dissimilarity measure (1-TOM). Finally, we identified 7 modules relevant to clinical traits, as eigengene adjacency heatmap descripted (Figure 4B). Of these, the turquoise module was most highly correlated with DEIncRNAs (Figure 4B,4C). Interestingly, we found that the correlation coefficient between royalblue module and progressive disease reached to 0.94 , indicating that the royalblue module is a gene set specifically associated with progression of disease. The royalblue module is also the most relevant module to distant metastasis (cor=0.72), indicating the correlation of IncRNAs with prognosis. By setting the module membership (MM) to $>0.8$ and the gene significance (GS) to $>0.4$ as threshold, we selected a total of 81 hub IncRNAs from the modules (Table S5), most of which were involved in the recurrence or metastasis of disease. The results indicated that the DEIncRNAs identified were involved in the prognosis of stage I LUAD patients.

Subsequently, we found that 19 genes were associated with distant metastasis, 5 genes associated with locoregional recurrence and 11 genes associated with distant metastasis, and another 65 genes associated with new primary tumor. Of the hub genes identified, FENDRR, LNC00639 and RP4-676L2.1 were also predicted as ceRNAs in stage I LUAD constructed by our database. Furthermore, we constructed ceRNA network using the three IncRNAs by Cytoscape (Figure 5A). Finally, ceRNA network containing 174 IncRNA-miRNA-mRNA regulatory relation for FENDRR, 937 IncRNA-miRNA-mRNA regulatory relation for 
$L N C 00639$, and 54 regulatory relation for $R P 4-676 L 2.1$. It showed that and $L N C 00639$ were hub nodes that could target more miRNAs and mRNAs in the network. Further, RT-PCR indicated that expression of FENDRR was significantly increased in LUAD tissues compared with paired normal tissues ( $P$ value $<$ .001) (Figure 5B), while the expression of RP4-676L2.1 and LNC00639 were not significant. Thus, the results revealed that FENDRR overexpression maybe involved in tumorigenesis.

To further explore the three IncRNAs identified, we evaluated their effects on recurrence by univariate Cox regression analysis in TCGA dataset. It showed that FENDRR was significantly associated with PFS (HRs $=1.69,95 \% \mathrm{Cl} 1.07-2.68, P<.05$ ), indicating that $F E N D R R$ could be regarded as a prognostic factor of tumor recurrence. Using median FPKM of FENDRR as cutoff value, we divided the patients into highexpression group and low-expression group. KM survival curve for the two groups indicated that PFS in the high-expression displayed significantly longer than that in the low-expression group (Figure 5C).

\section{Discussion}

Although it has achieved great improvement on clinical management for LUAD aided by the discovery of genetic mutations, there is still main obstacles on improving prognosis of patients resulting from its apt to migration and invasion. Therefore, the promise of utilization of IncRNAs in predicating clinical prognosis has attracted much attentions in translational research. In this study, we identify three IncRNAs, FENDRR, LNC00639 and RP4-676L2.1, which might be related with prognosis of stage I LUAD patients. However, only FENDRR is predicted to be associated with PFS of patients, and validated to be overexpressed in LUAD tissues by RT-PCR assay. The primary results uncover the potential role of FENDRR in the progression of early-stage LUAD. Importantly, the study gives a novel hint of the mechanism by which FENDRR might involve in the progression of disease.

Previous studies have revealed that IncRNAs could exert their biological function via interaction with DNA, RNA and protein depending on their location within cells[22]. Although there is still a lot to learn about IncRNA, accumulating studies demonstrated its function on relieving target mRNAs degradation mediated by miRNA, playing a role as ceRNAs, which could act as vital regulators in various physiological and pathological process of tumor [23,24]. To date, despite the plethora of reports, several IncRNAs have been identified to be involved in recurrence and metastasis of lung cancer [25-27]. In this study, we explored the most likely significant IncRNAs profile alternations of stage I LUAD, and revealed that FENDRR, LNC00639 and RP4-676L2.1 were predicted to be associated with progression of disease. However, only FENDRR were validated as predictive IncRNA of PFS by cox regression analysis, indicating its underlying function responsible for progression of LUAD.

LncRNA fetal-lethal non-coding developmental regulatory RNA (FENDRR), also named as FOXF1-AS1, is an intergenic IncRNA with consisting of seven exons. It is located at 3q13.31, 1,354 bp upstream of transcriptional start site, which is transcribed from a bidirectional promoter shared with the protein coding gene Foxf1 and Pitx2. Previous studies revealed that FENDRR could regulate cell migration, invasion, and lymphatic metastasis, demonstrating its inhibitory regulation in tumor progression[28]. It was reported 
that FENDRR was highly expressed in the lung, while was lowly expressed in the liver, colon and brain tissues, and the level of which was associated with prognosis of patients [29]. In breast cancer, the low level of FENDRR was associated with poor prognosis of patients, including a shorter survival and a shorter PFS [30]. Also, FENDRR was found to be related with survival of gastric cancer patients, the expression of which could be suppressed in gastric cancer associated fibroblasts by hypermethylation [31]. In agreement with previous studies[28], the level of FENDRR expression in tumor was also shown to be suppressed compared with paired normal tissue.

The mechanism of anti-malignant effects for FENDRR might involve in inhibiting cell migration, invasion and mediated stem-like properties by regulating epithelial mesenchymal transition (EMT) [32-34]. Previous studies reported that FENDRR could anchor PRC2 and/or TrxG/MLL complexes at its target promoters, increasing PRC2 occupancy and H2K27 trimethylation, which lead to the attenuation of target gene expression [35]. In gastric cancer, FENDRR was found to increase cell migration and invasion via upregulation of FN1, MMP2 and MMP9 [31]. In vitro, FENDRR was revealed to decrease the IC50 for cisplatin in A549/DDP cells, and depressed chemotherapy resistance to cisplatin in NSCLC [34]. Hitherto, the contribution of FENDRR on progression of LUAD has not been well clarified. In our dataset, FENDRR could targeted miR-6815-5p in ceRNA network constructed, suggesting that it might exert function on regulation expression of target mRNAs by 'sponging' miR-6815-5p. To the best our knowledge, this is the first study where predicts the regulation of miR-6815-5p on targeted mRNAs in LUAD. Apart from the predication of IncRNAs on PFS, the main breakthrough of our study is centred around the regulation of miR-6815-5p on FENDRR, which needs to explore its molecular mechanisms to increase further confidence to this result.

Regarding RP4-676L2.1 and $L N C 00639$, we speculated that they could be related with recurrence of disease by WGCNA using TCGA data set, suggesting their prognostic value in stage I LUAD. However, they were not significantly correlated with PFS of stage I LUAD patients by cox regression analysis.

Surprisingly, RP4-676L2.1 and LNC00639 were also not successfully validated to be consistent difference of expression between tumor and normal lung tissue by qPCR. We speculated that it may lie with the bioinformatic analysis. Still, further studies are needed to validate using larger samples.

The discovery of IncRNAs contributes significantly to clinical prognosis in stage I LUAD patients. Its strength lies in not only testing using the sequencing dataset, but also validating using TCGA dataset by bioinformatics methods. Still, some limitations must be noted. Firstly, the ceRNAs network were predicted by bioinformatics methods, which needs to be validated by functional experiments further. Secondly, due to the low number of samples performed RNA-seq, the WGCNA was constructed using TCGA dataset, which might induce racial bias. This is not surprising, a substantial number of studies on IncRNAs have been validated using TCGA dataset. Thirdly, the IncRNAs identified from RNA-seq were inconsistent with the qPCR results, which may result from incorrect annotation in the bioinformatic analysis.

\section{Conclusions}


Taken together, this study contributed significantly to the wider knowledge of IncRNAs and ceRNAs involvement of progression of stage I LUAD. Out of the three IncRNAs validated, FENDRR is validated to involve in the recurrence of disease, which confirm previous findings. Based on the FENDRR-related ceRNA network constructed, it was revealed the regulation of miR-6815-5p on FENDRR for the first time, therefore open to further research to explore molecular mechanism. However, studies are still needed to establish the role of FENDRR/miR-6815-5p axis in the progression of early-stage LUAD.

\section{Abbreviations}

IncRNAs: long non-coding RNAs; NSCLC: Non-small cell lung cancer; LUAD: Lung adenocarcinoma; ceRNA: competing endogenous RNA; PFS: progression free survival; CBS: cystathionine- $\beta$-synthase; RIG: retinoid-inducible protein; TCGA: The Cancer Genome Atlas; DElncRNAs: identify differentially expressed IncRNAs; KEGG: Kyoto Encyclopedia of Genes and Genomes; BP: biological processes; CC: cellular component; MF: molecular function; WGCNA: Weighted gene correlation network analysis; KM: KaplanMeier; HR: hazard ratio; FENDRR: fetal-lethal non-coding developmental regulatory RNA;

\section{Declarations}

\section{Acknowledgements}

Not applicable.

\section{Authors' contributions}

$X Y, L G U, L Y F, L X B$ and ZYM participated in the study design. XY, LGF and LYF performed the most of the RNA-seq analysis and drafted the paper. LYF and LXB performed the WGCNA. LH, GZF and XYX participated in the design of figures. LXB, CSH and YJS participated in the acquisition of LUAD tissues. All authors read and approved the final manuscript.

\section{Funding}

The work was supported by Fujian Provincial Health Fund for Young and Middle-aged People (2019ZQNB-7) and Quanzhou major science and technology projects (2018-QDZX-9).

\section{Availability of data and materials}

The datasets used and/or analyzed during the current study are available from the corresponding author on reasonable request. We will deposit the sequencing data of this study in the NCBI SRA database after accepting the article.

\section{Ethics approval and consent to participate}


The ethics committee of The Second Affiliated Hospital of Fujian Medical University approved this study and is in compliance with the Helsinki Declaration. Written informed consent was obtained from all individual participants included in the study.

\section{Consent for publication}

Not applicable.

\section{Competing interests}

The authors declare that they have no competing interests.

\section{References}

1. Bray F, Ferlay J, Soerjomataram I, Siegel RL, Torre LA, Jemal A: Global cancer statistics 2018: GLOBOCAN estimates of incidence and mortality worldwide for 36 cancers in 185 countries. CA Cancer J Clin 2018, 68(6):394-424. https://doi.org/10.3322/caac.21492

2. Siegel RL, Miller KD, Jemal A: Cancer statistics, 2020. CA Cancer J Clin 2020, 70(1):7-30. https://doi.org/10.3322/caac. 21590

3. Goldstraw P, Chansky K, Crowley J, Rami-Porta R, Asamura H, Eberhardt WE, Nicholson AG, Groome P, Mitchell A, Bolejack V et al: The IASLC Lung Cancer Staging Project: Proposals for Revision of the TNM Stage Groupings in the Forthcoming (Eighth) Edition of the TNM Classification for Lung Cancer. J Thorac Oncol 2016, 11(1):39-51. https://doi.org/10.1016/j.jtho.2015.09.009

4. Herbst R, Morgensztern D, Boshoff CJN: The biology and management of non-small cell lung cancer. 2018, 553(7689):446-454. https://doi.org/10.1038/nature25183

5. Hung J, Hsu W, Hsieh C, Huang B, Huang M, Liu J, Wu YJT: Post-recurrence survival in completely resected stage I non-small cell lung cancer with local recurrence. 2009, 64(3):192-196. https://doi.org/10.1136/thx.2007.094912

6. Spiro SG, Tanner NT, Silvestri GA, Janes SM, Lim E, Vansteenkiste JF, Pirker R: Lung cancer: progress in diagnosis, staging and therapy. Respirology 2010, 15(1):44-50. https://doi.org/10.1111/j.14401843.2009.01674.x

7. Guerrera F, Errico L, Evangelista A, Filosso P, Ruffini E, Lisi E, Bora G, Asteggiano E, Olivetti S, Lausi P et al: Exploring Stage I non-small-cell lung cancer: development of a prognostic model predicting 5year survival after surgical resectiont. 2015, 47(6):1037-1043. https://doi.org/10.1093/ejcts/ezu410

8. Rinn J, Chang HJArob: Genome regulation by long noncoding RNAs. 2012, 81:145-166. https://doi.org/10.1146/annurev-biochem-051410-092902

9. Cabili M, Trapnell C, Goff L, Koziol M, Tazon-Vega B, Regev A, Rinn JJG, development: Integrative annotation of human large intergenic noncoding RNAs reveals global properties and specific subclasses. 2011, 25(18):1915-1927. https://doi.org/10.1101/gad.17446611 
10. Geisler S, Coller JJNrMcb: RNA in unexpected places: long non-coding RNA functions in diverse cellular contexts. 2013, 14(11):699-712. https://doi.org/10.1038/nrm3679

11. Weidle U, Birzele F, Kollmorgen G, Rüger RJCg, proteomics: Long Non-coding RNAs and their Role in Metastasis. 2017, 14(3):143-160. https://doi.org/10.21873/cgp.20027

12. Karreth F, Tay Y, Perna D, Ala U, Tan S, Rust A, DeNicola G, Webster K, Weiss D, Perez-Mancera P et al: In vivo identification of tumor- suppressive PTEN ceRNAs in an oncogenic BRAF-induced mouse model of melanoma. 2011, 147(2):382-395. https://doi.org/10.1016/j.cell.2011.09.032

13. Cai J, Fang L, Huang Y, Li R, Xu X, Hu Z, Zhang L, Yang Y, Zhu X, Zhang H et al: Simultaneous overactivation of Wnt/ $\beta$-catenin and TGF $\beta$ signalling by miR-128-3p confers chemoresistanceassociated metastasis in NSCLC. 2017, 8:15870. https://doi.org/10.1038/ncomms15870

14. Yuan Y, Liao H, Pu Q, Ke X, Hu X, Ma Y, Luo X, Jiang Q, Gong Y, Wu M et al: miR-410 induces both epithelial-mesenchymal transition and radioresistance through activation of the $\mathrm{PI} 3 \mathrm{~K} / \mathrm{mTOR}$ pathway in non-small cell lung cancer. 2020, 5(1):85. https://doi.org/10.1038/s41392-020-0182-2

15. Y F, W X, W Z, W W, T L, X Z: LncRNA DCRF regulates cardiomyocyte autophagy by targeting miR$551 b-5 p$ in diabetic cardiomyopathy. \%J Theranostics. 2019, 9(15):4558-4566. https://doi.org/10.7150/thno.31052

16. Tang XD, Zhang DD, Jia L, Ji W, Zhao YS: IncRNA AFAP1-AS1 Promotes Migration and Invasion of Non-Small Cell Lung Cancer via Up-Regulating IRF7 and the RIG-I-Like Receptor Signaling Pathway. Cell Physiol Biochem 2018, 50(1):179-195. https://doi.org/10.1159/000493967

17. Pasquali S, Chiswell K, Hall M, Thibault D, Romano J, Gaynor J, Shahian D, Jacobs M, Gaies M, O'Brien S et al: Estimating Resource Utilization in Congenital Heart Surgery. 2020, 110(3):962-968. https://doi.org/10.1016/j.athoracsur.2020.01.013

18. Kadota K, Sima C, Arcila M, Hedvat C, Kris M, Jones D, Adusumilli P, Travis WJTAjosp: KRAS Mutation Is a Significant Prognostic Factor in Early-stage Lung Adenocarcinoma. 2016, 40(12):1579-1590. https://doi.org/10.1097/pas.0000000000000744

19. Sumazin P, Yang X, Chiu H, Chung W, lyer A, Llobet-Navas D, Rajbhandari P, Bansal M, Guarnieri P, Silva $\mathrm{J}$ et al: An extensive microRNA-mediated network of RNA-RNA interactions regulates established oncogenic pathways in glioblastoma. 2011, 147(2):370-381. https://doi.org/10.1016/j.cell.2011.09.041

20. Langfelder P, Horvath SJBb: WGCNA: an R package for weighted correlation network analysis. 2008, 9:559. https://doi.org/10.1186/1471-2105-9-559

21. Langfelder P, Horvath SJJoss: Fast R Functions for Robust Correlations and Hierarchical Clustering. 2012, 46(11)

22. Chen C, He W, Huang J, Wang B, Li H, Cai Q, Su F, Bi J, Liu H, Zhang B et al: LNMAT1 promotes lymphatic metastasis of bladder cancer via CCL2 dependent macrophage recruitment. 2018, 9(1):3826. https://doi.org/10.1038/s41467-018-06152-x 
23. Xu J, Meng Q, Li X, Yang H, Xu J, Gao N, Sun H, Wu S, Familiari G, Relucenti M et al: Long Noncoding RNA MIR17HG Promotes Colorectal Cancer Progression via miR-17-5p. 2019, 79(19):4882-4895. https://doi.org/10.1158/0008-5472.Can-18-3880

24. Yu W, Ding J, He M, Chen Y, Wang R, Han Z, Xing E, Zhang C, Yeh SJO: Estrogen receptor $\beta$ promotes the vasculogenic mimicry (VM) and cell invasion via altering the IncRNA-MALAT1/miR-1455p/NEDD9 signals in lung cancer. 2019, 38(8):1225-1238. https://doi.org/10.1038/s41388-0180463-1

25. Yuan S, Xiang Y, Wang G, Zhou M, Meng G, Liu Q, Hu Z, Li C, Xie W, Wu N et al: Hypoxia-sensitive LINC01436 is regulated by E2F6 and acts as an oncogene by targeting miR-30a-3p in non-small cell lung cancer. 2019, 13(4):840-856. https://doi.org/10.1002/1878-0261.12437

26. Wu D, Yang B, Chen J, Xiong H, Li Y, Pan Z, Cao Y, Chen J, Li T, Zhou S et al: Upregulation of long noncoding RNA RAB1A-2 induces FGF1 expression worsening lung cancer prognosis. 2018, 438:116125. https://doi.org/10.1016/j.canlet.2018.09.016

27. Li C, Wan L, Liu Z, Xu G, Wang S, Su Z, Zhang Y, Zhang C, Liu X, Lei Z et al: Long non-coding RNA XIST promotes TGF- $\beta$-induced epithelial-mesenchymal transition by regulating miR-367/141-ZEB2 axis in non-small-cell lung cancer. 2018, 418:185-195. https://doi.org/10.1016/j.canlet.2018.01.036

28. Acha-Sagredo A, Uko B, Pantazi P, Bediaga NG, Moschandrea C, Rainbow L, Marcus MW, Davies MPA, Field JK, Liloglou T: Long non-coding RNA dysregulation is a frequent event in non-small cell lung carcinoma pathogenesis. Br J Cancer 2020, 122(7):1050-1058. https://doi.org/10.1038/s41416-0200742-9

29. Sauvageau M, Goff L, Lodato S, Bonev B, Groff A, Gerhardinger C, Sanchez-Gomez D, Hacisuleyman E, Li E, Spence M et al: Multiple knockout mouse models reveal lincRNAs are required for life and brain development. 2013, 2:e01749. https://doi.org/10.7554/eLife.01749

30. Li Y, Zhang W, Liu P, Xu Y, Tang L, Chen W, Guan XJO, therapy: Long non-coding RNA FENDRR inhibits cell proliferation and is associated with good prognosis in breast cancer. 2018, 11:1403-1412. https://doi.org/10.2147/ott.S149511

31. Xu T, Huang M, Xia R, Liu X, Sun M, Yin L, Chen W, Han L, Zhang E, Kong R et al: Decreased expression of the long non-coding RNA FENDRR is associated with poor prognosis in gastric cancer and FENDRR regulates gastric cancer cell metastasis by affecting fibronectin1 expression. 2014, 7:63. https://doi.org/10.1186/s13045-014-0063-7

32. Wei H, Nickoloff J, Chen W, Liu H, Lo W, Chang Y, Yang P, Wu C, Williams D, Gelovani J et al: FOXF1 mediates mesenchymal stem cell fusion-induced reprogramming of lung cancer cells. 2014, 5(19):9514-9529. https://doi.org/10.18632/oncotarget.2413

33. Miao L, Huang Z, Zengli Z, Li H, Chen Q, Yao C, Cai H, Xiao Y, Xia H, Wang YJO: Loss of long noncoding RNA FOXF1-AS1 regulates epithelial-mesenchymal transition, stemness and metastasis of non-small cell lung cancer cells. 2016, 7(42):68339-68349. https://doi.org/10.18632/oncotarget.11630 
34. Kun-Peng Z, Chun-Lin Z, Xiao-Long MJljobs: Antisense IncRNA FOXF1-AS1 Promotes Migration and Invasion of Osteosarcoma Cells Through the FOXF1/MMP-2/-9 Pathway. 2017, 13(9):1180-1191. https://doi.org/10.7150/ijbs.21722

35. Grote P, Wittler L, Hendrix D, Koch F, Währisch S, Beisaw A, Macura K, Bläss G, Kellis M, Werber M et al: The tissue-specific IncRNA Fendrr is an essential regulator of heart and body wall development in the mouse. 2013, 24(2):206-214. https://doi.org/10.1016/j.devcel.2012.12.012

\section{Figures}




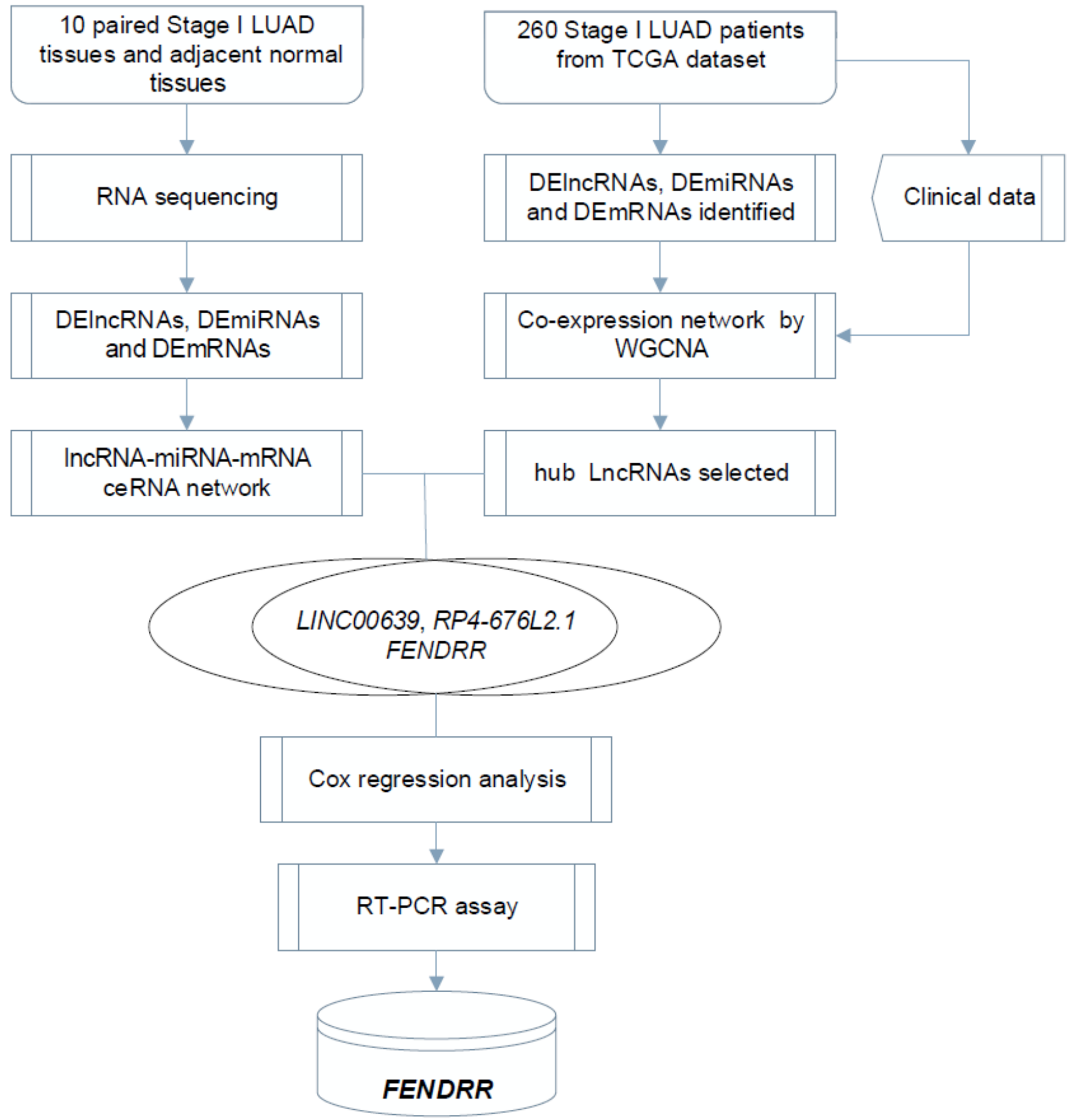

Figure 1

The flowchart of the present study. DElncRNAs: differentially expressed IncRNA; DEmiRNAs: differentially expressed miRNA; DEmRNAs: differentially expressed mRNA; WGCNA: Weighted gene correlation network analysis. 
A

B

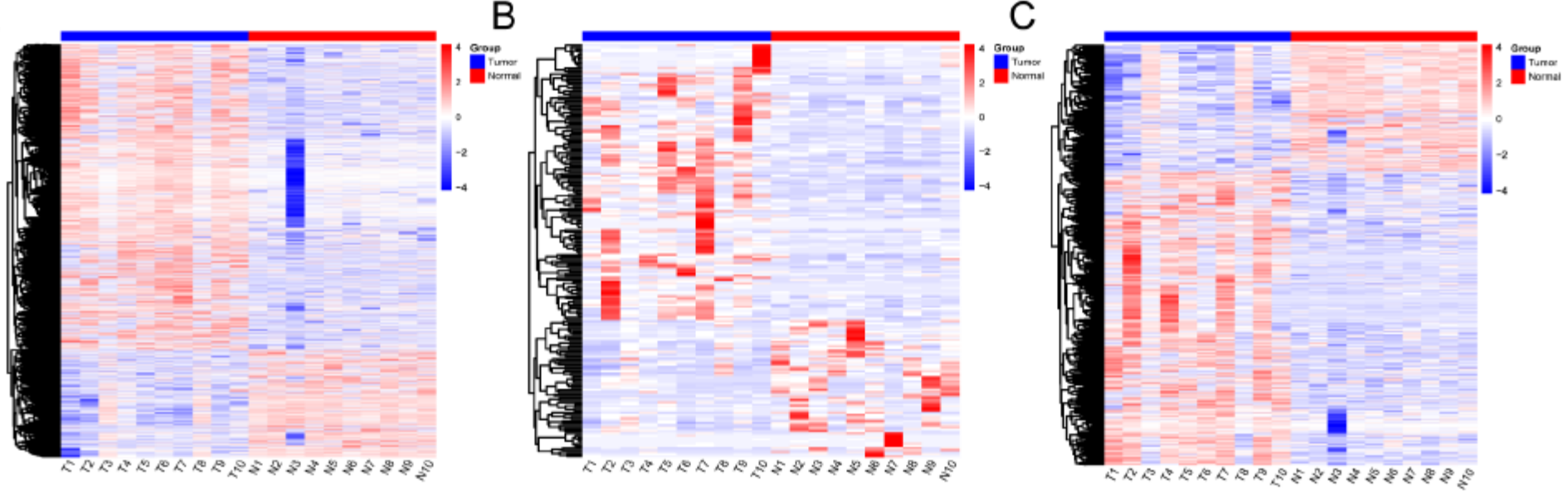

D

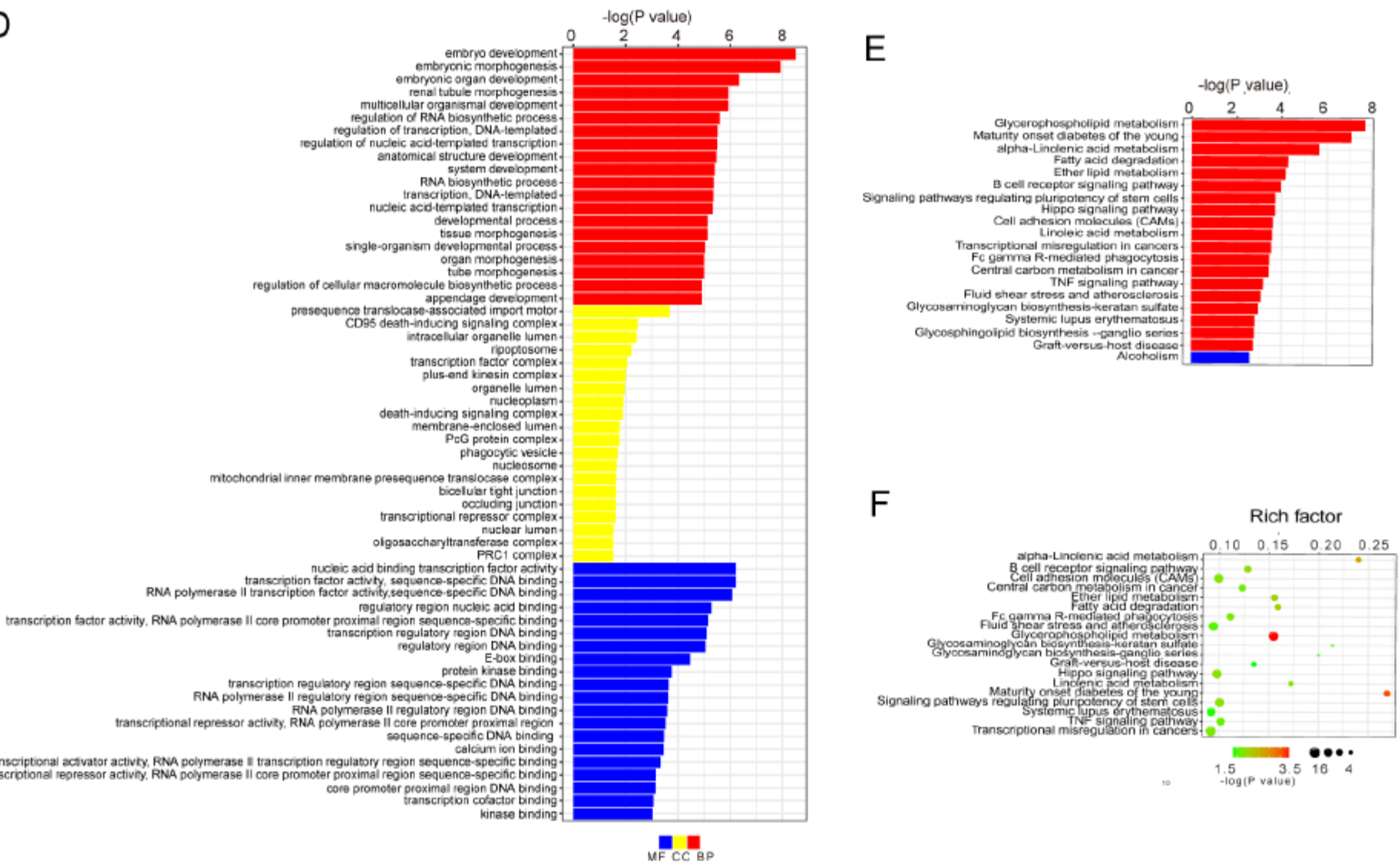

Figure 2

The heatmaps of DEmRNAs, DEmiRNAs and DElncRNAs in the expression profile in 10 paired LUAD and adjacent normal tissue (Figure 2A, B, C, respectively). Top $20 \mathrm{GO}$ terms enriched in DElncRNAs (Figure 2D). Top 20 pathways enriched in DElncRNAs using Kyoto Encyclopedia of Genes and Genomes (KEGG) analysis (Figure 2E) and displayed in bubble chart (Figure 2F). BP: biological processes; CC: cellular component; MF: molecular function. 

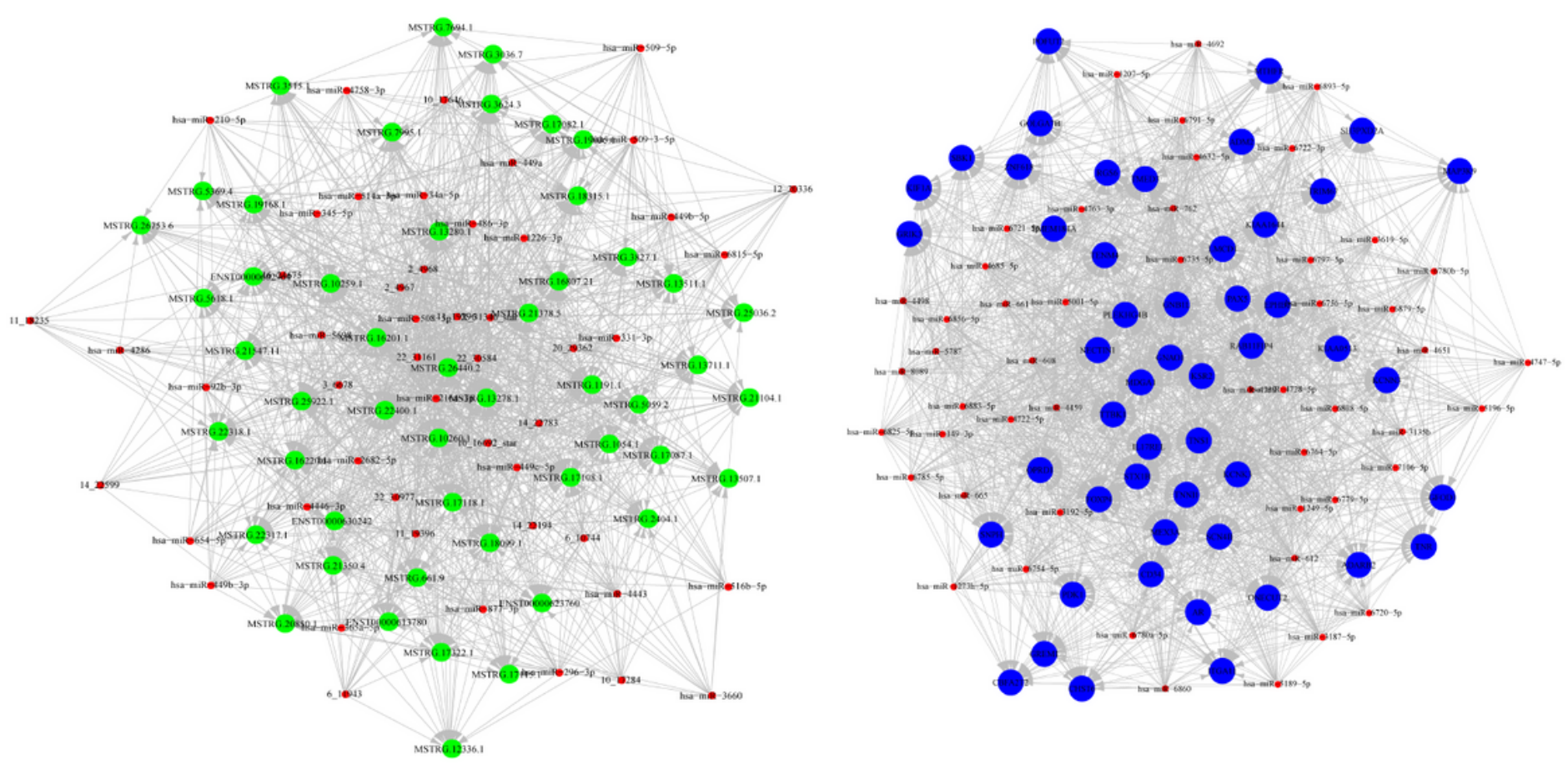

Figure 3

The regulation relationship between miRNA and mRNA predicted by miRanda (Figure $3 \mathrm{~A}$ ), and the relationship between miRNA and IncRNA predicted by miRanda (Figure 3B), with the criteria of total score $\geq 150$ and total energy $\leq-20$. The green node represents IncRNAs; the red node represents miRNAs; the blue node represents mRNAs. 
a

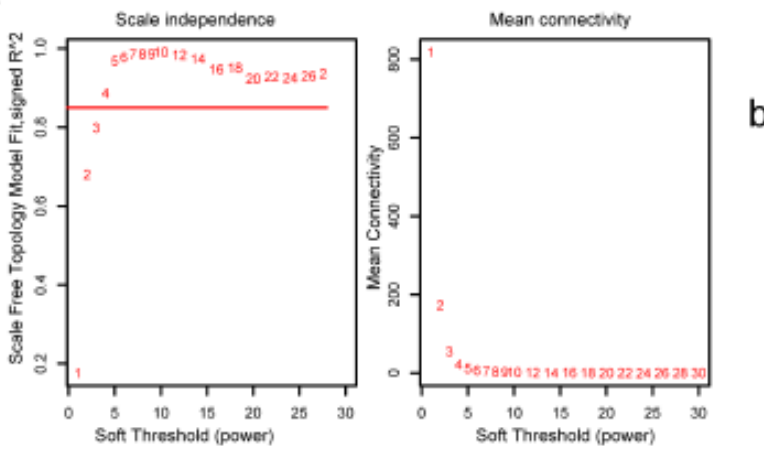

b

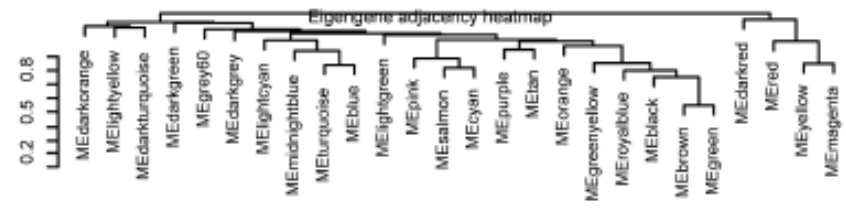

C
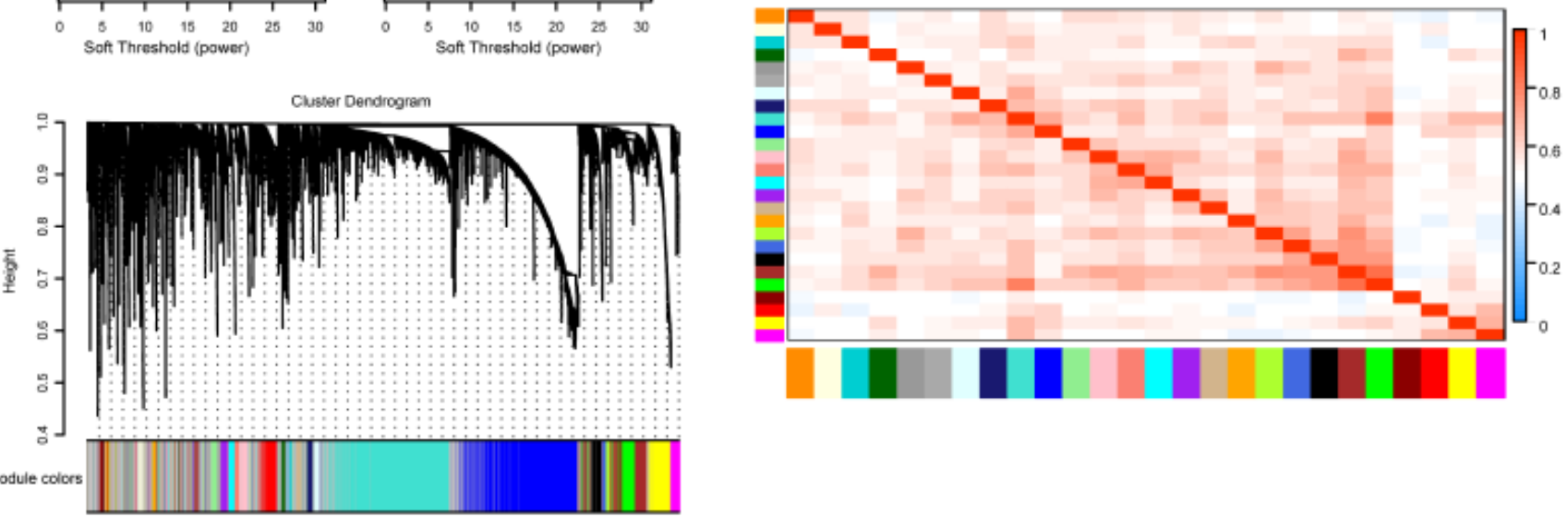

d

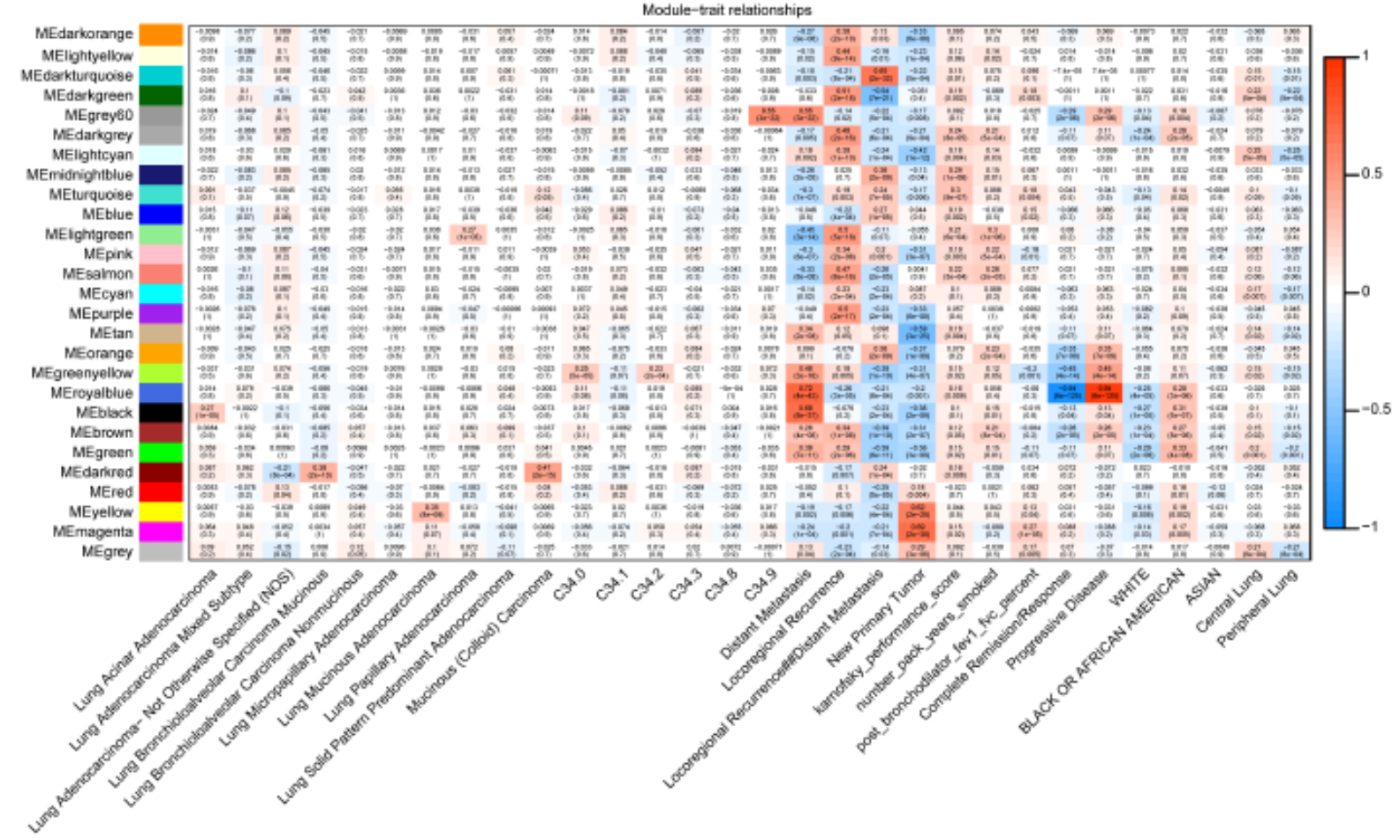

Figure 4

WGCNA and identification of significant modules. (A) The soft-thresholding power in WGCNA. (B) The eigengene of each colored module were calculated to establish an adjacent matrix. (C) Cluster dendrogram obtained from IncRNAs data of stage I LUAD in TCGA dataset with average hierarchical linkage clustering. The color row underneath the dendrogram represents the module assigned by 
Dynamic Tree Cut. (D) Module-trait relationship heatmap. The row represents the modules, while the column represents the trait. The values in the box represents the correlation and $\mathrm{P}$ values.

A

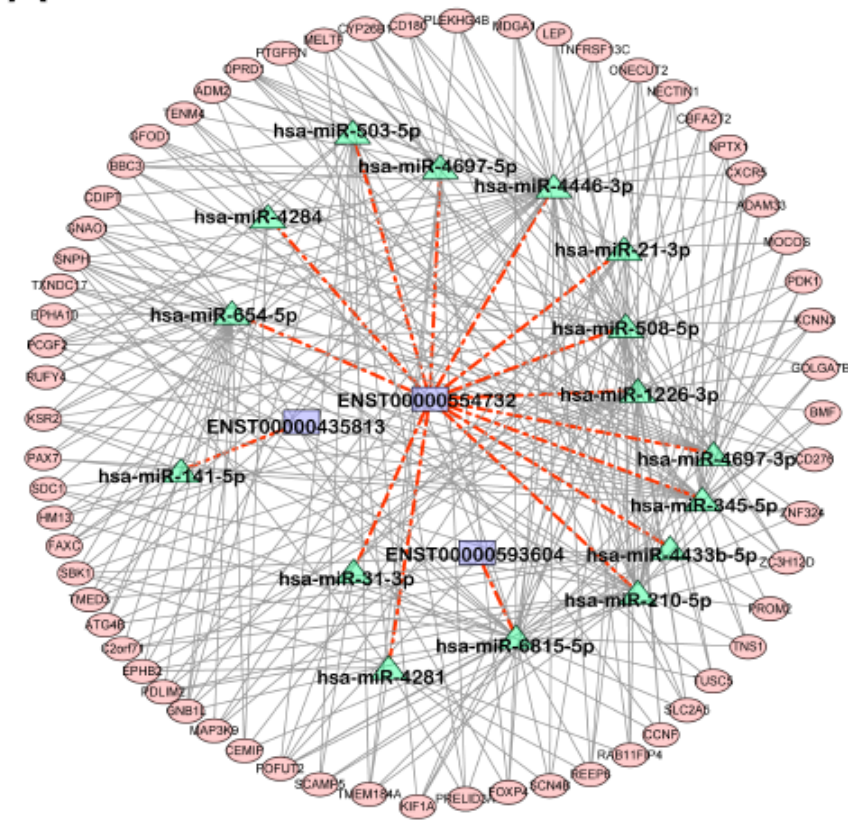

B

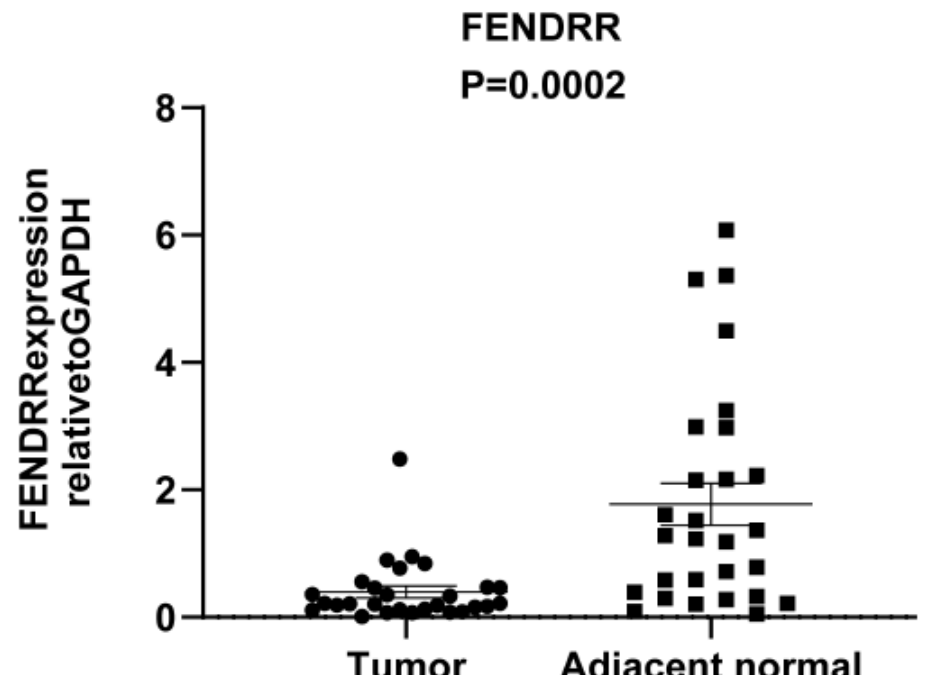

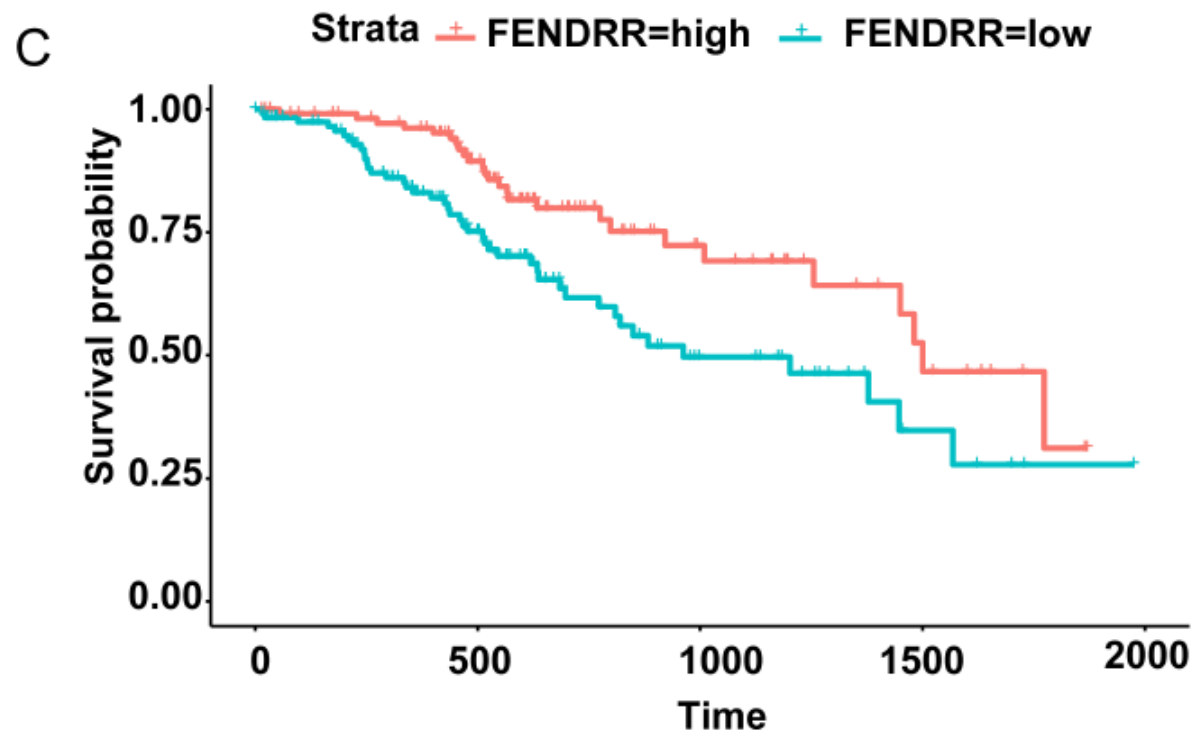

Figure 5

The ceRNA network constructed by FENDRR, LNC00639 and RP4-676L2.1 (Figure 5A). The FENDRR expression levels of stage I LUAD tissues and paired normal tissues were tested by RT-PCR $(n=28$, Figure $5 B)$. Prognostic significance of FENDRR expression on PFS for stage I LUAD patients by the median value as cutoff (Figure 5C).

\section{Supplementary Files}

This is a list of supplementary files associated with this preprint. Click to download. 
- Additionalfile1TableS1S5.docx

- Additionalfile2Figures1.pdf 